\title{
Ophthalmia neonatorum: study of a decade of experience at the Mount Sinai Hospital
}

\author{
VINCENT N JARVIS,' REBECCA LEVINE, ' AND PENNY A ASBELL' \\ From the Departments of 'Ophthalmology and 'Biomathematical Sciences, Mount Sinai School of Medicine, \\ 1 Gustave L Levy Place, New York, New York 10029, USA
}

SUMMARY We reviewed the charts of neonates who received a diagnosis of conjunctivitis or ophthalmia neonatorum during a 10-year period at the Mount Sinai Hospital, New York City. Ninety-five cases were discovered, four of these were born elsewhere and were not subject to our protocol, which at present consists of intramuscular penicillin and topical tetracycline ointment $(1 \%)$. The 91 cases represent an incidence of $3 \cdot 1$ cases per 1000 live births $(9 \cdot 1 /$ year $)$, an incidence approximately equal to that reported in other published studies. The clinical characteristics examined were: age, sex, race, birth weight, gestational age, Apgar scores, presence or absence of fever or other systemic illnesses, complications, type of delivery, time of year, incubation period, presence and type of discharge, uni- or bilaterality, Gram stain, Giemsa stain, culture results, antibiotic disc sensitivities, cervical culture, antibiotic therapy, sequelae, and type of prophylaxis received. Seven aetiological diagnostic categories were established: gonococcal (3\%), chlamydial $(3 \%)$, staphylococcal $(30 \%)$, other pathogens $(25 \%)$, chemical conjunctivitis $(7 \%)$, culture negative/normal flora $(22 \%)$, and unobtainable $(10 \%)$. This distribution differs from others published. In contrast with other studies, moreover, we found few gonococcal and no chlamydial cases in neonates subject to the Mount Sinai Hospital treatment protocol. Correlation of clinical characteristics and aetiological categories showed no statistically significant trend. So far there have been no cases of ophthalmia neonatorum, treated as on our protocol, caused by penicillin resistant gonococci or chlamydiae.

Ophthalmia neonatorum, that is, conjunctivitis occurring within the first four weeks of life, has a notable history and was once a major cause of neonatal blindness. The syndrome has been associated with numerous organisms, which have varied in their relative importance over time.'

Clinicians are well aware that neonates very often develop a 'red eye' within the first few days of life. This is usually caused by trauma, chemical irritation, or transient infection. However, more serious forms of conjunctivitis do occur, and various methods of prophylaxis have been employed over the years to prevent the sequelae of the more serious pathogenic processes.

For over a hundred years Credé's instillation of silver nitrate solution has generally been the primary mode of prevention of gonococcal ophthalmia neonatorum. Silver nitrate, however, has many negative Correspondence to Dr P A Asbell. characteristics: (1) variable efficacy against modern strains of gonococci; (2) no efficacy against chlamydia (which may be the leading cause of ophthalmia neonatorum); (3) the possible induction of severe conjunctivitis by the solution itself; (4) blindness from accidental overdosing; and (5) the loss of efficacy from the postadministration saline wash. ${ }^{2}$

The Mount Sinai Hospital (MSH), New York City, introduced in 1952 the injection of 50000 units of aqueous penicillin intramuscularly (IM) to all liveborn infants in place of silver nitrate. So far we have had no immediate or short-term sensitivity problems with penicillin. ${ }^{3}$ In 1982 a $1 \%$ tetracycline ointment instillation was added to this protocol.

The purpose of this retrospective study is to examine these prophylactic measures and the causes, incidence, clinical features, and treatment of ophthalmia neonatorum at MSH for the past 10 years. 


\section{Materials and methods}

The charts of all neonates (0-28 days) delivered at or presenting to MSH between June 1973 and June 1983 who received a diagnosis of conjunctivitis or ophthalmia neonatorum were reviewed. Clinical characteristics were recorded on a standardised clinical form and included: infant's age, sex, race, birth weight, gestational age, Apgar scores, presence or absence of fever, other systemic illnesses, complications and type of delivery, month of diagnosis, incubation period, presence and type of discharge, uni- or bilaterality, Gram stain, Giemsa stain, culture results, antibiotic disc sensitivities (where appropriate), cervical culture, antibiotic therapy, sequelae, and type of prophylaxis received.

The statistical analysis of data was by Pearson's $\chi^{2}$ test and one-way analysis of variance. The analysis was performed on the actual numbers.

\section{Results}

Ninety-eight neonates received a diagnosis of ophthalmia neonatorum during the 10 -year period. Three charts were unretrievable, and only 95 cases are presented here. Four of these were born elsewhere and were therefore subject to different prophylactic measures. During the study period a total of 30345 live births were recorded at MSH. Undoubtedly some cases of ophthalmia neonatorum escaped detection because of the mild nature of the process and the possibility of neonates born here presenting elsewhere. Therefore this series represents a minimal incidence of $3 \cdot 1$ cases per 1000 live births, with an average of 9.5 cases per year, including those infants born elsewhere. Apart from those our series yields a similar incidence, with an average of $9 \cdot 1$ cases per year.

\section{AETIOLOGY}

Seven diagnostic categories were established by aetiology (see Table 1 for percentages and abbreviations).

The diagnosis of GON was made in three cases, representing $3 \cdot 1 \%$ of all ophthalmia neonatorum cases in our study. The most recent was a case of a $2640 \mathrm{~g}$ boy born by an uncomplicated spontaneous vaginal delvery at MSH. He received 50000 units of penicillin $\mathrm{G}$ intramuscularly at birth. He was a fullterm neonate with Apgar scores of 7 (1 minute) and 8 (5 minutes) and had no systemic symptoms. A mild unilateral eye discharge developed on the third day of life, but a swab of conjunctival exudate sent to the microbiology laboratory in transport media grew only Staphylococcus epidermidis. Despite prolonged treatment with sulphisoxazole ophthalmic drops, scant mucus persisted in both eyes. He presented with ophthalmia neonatorum on day 19 of life.

Two months prior to the birth the father had visited Acapulco, Mexico. After his return to New York City the parents had sexual relations. The mother noted a new vaginal discharge one week before the delivery.

The infant was treated with penicillin G $(100000$ units/kg/day) intravenously. Local therapy consisted of frequent saline lavage followed by topical penicillin drops $(100000 \mathrm{U} / \mathrm{ml})$. The eyes improved markedly within 12 hours, although subsequent improvement was slower than expected. Culture of the conjunctiva grew $N$. gonorrhoeae. The organism produced a $\beta$-lactamase and was resistant to penicillin (MIC greater than $20 \mu \mathrm{g} / \mathrm{ml}$ ). Cure was achieved with parenteral cephazolin (to which the isolate was sensitive) and topical gentamicin eyedrops. $^{4}$

The second case was less certain but highly suggestive of GON. A full-term $2620 \mathrm{~g}$ girl was born vaginally with mid-forceps assistance due to slow progression of labour. Also of note during delivery was the premature rupture of the amniotic membrane more than 24 hours before birth. The neonate received 50000 units of penicillin $G$ prophylactically at birth but presented immediately with bilateral, purulent eye discharge and fever. Apgar scores were 8 at 1 minute and 10 at 5 minutes. The chart reviewed contained no record of Gram stain results, and eye cultures were negative. However, a gastric aspirate of the neonate was culture positive for $N$. gonorrhoeae, which was penicillin-sensitive. The mother had a history of partially treated gonorrhoea within six months of delivery. The infant was treated with penicillin $\mathrm{G}(100000 \mathrm{U} / \mathrm{kg} / \mathrm{day})$ and gentamicin $(6 \mathrm{mg} / \mathrm{kg} / \mathrm{day})$ intravenously. Both mother and child were discharged without complications. The followup examination was within normal limits.

The third case was one of a mixed infection described below.

Chlamydia can be demonstrated by one of three laboratory methods: (1) identification of typical

Table 1 Aetiology of 95 cases of ophthalmia neonatorum*

\begin{tabular}{lrr}
\hline Aetiology & No. & $\%$ \\
\hline Gonococcal (GON) $\dagger$ & 3 & $3 \cdot 1$ \\
Chlamydial (ChON) $\dagger$ & 3 & $3 \cdot 1$ \\
Staphylococcal (SON) & 29 & $30 \cdot 2$ \\
Other pathogens (OON) & 24 & $25 \cdot 3$ \\
Chemical (CC) & 7 & $7 \cdot 4$ \\
Culture negative/normal flora (NON) & 21 & $22 \cdot 1$ \\
Unobtainable (UON) & 9 & $9 \cdot 4$ \\
\hline
\end{tabular}

*Includes four cases born elsewhere.

†Includes one mixed infection (gonococcal and chlamydial). 
Table 2 Results of conjunctival cultures in 24 cases of ophthalmia neonatorum due to other pathogens (OON)

\begin{tabular}{ll}
\hline Culture results & No. of cases \\
\hline Escherichia coli & 9 \\
Str. viridans & 4 \\
Str. fecalis & 2 \\
Str. pneumoniae & 2 \\
Staph. aureus and E. coli & 2 \\
Staph. aureus and Pseudomonas aeruginosa & 1 \\
Serratia & 1 \\
Klebsiella oxytoca & 1 \\
Enterococcus & 1 \\
Haemophilus parainfluenzae & 1 \\
\hline
\end{tabular}

Halberstadten-Prowazek inclusion bodies from conjunctival scrapings by means of iodine, Giemsa, or fluorescent antibody staining; (2) complement fixation or microtitre indirect immunofluorescence techniques; or (3) direct growth of the organism in embryonated hens' eggs or tissue culture systems. ${ }^{5}$

In this study, where conjunctival scrapings were obtained, prepared, examined, and noted in the charts by the house staff, method number one (1) above was employed as the diagnostic criterion for chlamydial ON (ChON).

Three cases of ChON were found, representing $3.1 \%$ of all cases of ophthalmia neonatorum in this series. Two of these had chart notations by house staff describing the inclusion bodies on Giemsa stain. The third case also had the bodies but was unique in that it was a mixed infection.

The mixed infection occurred in a $2920 \mathrm{~g}$ Hispanic boy who was the full-term product of a normal spontaneous vaginal delivery performed at another hospital where he had received silver nitrate prophylaxis. He presented to the MSH emergency room on the fourth day of life with a three-day history of a yellow, mucoid discharge from both eyes. Gram staining of scrapings revealed numerous polymorphonuclear leucocytes and Gram-negative diplococci morphologically typical of gonococci. Intravenous penicillin and eyedrops with saline irrigation were begun, with dramatic improvement. However, on day eight of life, while he was still receiving hospital treatment, bilateral conjunctivitis again developed. Gram staining now showed neutrophils and mononuclear cells but no bacteria and a repeat culture showed no growth. A Giemsa stain of conjunctival scrapings revealed epithelial cells with nuclear capping and intracytoplasmic inclusion bodies. The penicillin was stopped and the child was cured with sulphacetamide eyedrops. ${ }^{\circ}$

The next diagnostic category, staphylococcal ophthalmia neonatorum (SON), comprises those cases in which only Staphylococcus aureus was identified by standard bacteriological techniques, namely, growth on blood agar, coagulase positivity, and typical colonial morphology. The isolates were then subject to antibiotic disc sensitivity screening. Staph. aureus was the most frequent isolate in this study, found in $30 \cdot 2 \%$ of all cases.

The category of 'other pathogens' (OON) consisted of 24 cases, representing $25 \cdot 3 \%$ of all cases of ophthalmia neonatorum. Organisms isolated were subject to antibiotic disc sensitivity screening and are listed on Table 2.

Chemical conjunctivitis (CC) was diagnosed in seven cases $(7 \cdot 4 \%)$ and comprised those cases in which symptoms appeared within 36 hours of birth and in which smears, Gram stains, and cultures were negative or showed only a normal flora, that is, Staph. epidermidis and diphtheroids.

The culture negative/normal flora group (NON) represented those cases in which symptoms began after 36 hours, the Gram stain was unremarkable, and cultures were negative for growth or grew only normal flora. Twenty-one cases $(22 \cdot 1 \%)$ belonged to this category. Obviously there may be some overlap between the NON and CC groups as the two differed only temporally.

The records of the final group were either unobtainable or no record of cultures could be found in the chart or in culture report log books. Nine cases $(9 \cdot 4 \%)$ fell into this category (UON).

\section{CLINICAL CHARACTERISTICS}

The clinical data on our standardised chart review form were tabulated and compared for the aetiological categories. No statistically significant trend was demonstrated by $\chi^{2}$ and, where appropriate, analysis of variance tests.

Of note is the fact that phototherapy treatment of the neonate did not produce any difference among the groups. Nor did premature rupture of membranes seem to predispose the infant to any of the established aetiological categories. However, for both the phototherapy exposed infants and for those who suffered premature rupture of membranes the absolute numbers were not sufficient to employ statistical analysis. In addition, according to the charts reviewed, no cervical cultures were performed and no sequelae were noted in any of the infants seen at follow-up.

Examination of monthly incidences revealed no monthly trends or seasonal variations when all seven categories were grouped. Also, if CC, NON, and UON categories are analysed as one group, again no trends or variations are evident. Hence, viral aetiology is unlikely.

The CC group showed no significant association with premature rupture of membranes, meconium 
Table 3 Ophthalmia neonatorum prophylaxis comparison at Mount Sinai Hospital*

\begin{tabular}{lcc}
\hline & $\begin{array}{l}\text { IM penicillin } \\
\text { only }\end{array}$ & $\begin{array}{l}\text { IM penicillin and } \\
\text { topical tetracycline }\end{array}$ \\
\hline Number of cases & 85 & 6 \\
Number of live births & 25845 & 4500 \\
Incidence cases per 100 live & $3 \cdot 33$ & $1 \cdot 33$ \\
$\quad$ births & & \\
Number of cases by aetiology & 2 & 0 \\
GON & 0 & 0 \\
ChON & 27 & 1 \\
SON & 22 & 2 \\
OON & 9 & 0 \\
UON & 5 & 2 \\
CC & 20 & 1 \\
NON & & \\
\hline
\end{tabular}

${ }^{*}$ Excludes four cases born elsewhere.

present at birth, type of delivery, or with tetracycline ointment instillation as prophylaxis.

Of interest is the fact that all ChON cases in our study were born elsewhere and thereby subject to other prophylaxis. No cases of $\mathrm{ChON}$ were reported at MSH under either of our protocols. Furthermore no cases of GON have occurred at MSH since IM penicillin and topical tetracycline have been given as prophylaxis.

As stated earlier, the type of prophylaxis employed at the MSH has recently changed. A comparison of the two protocols is outlined on Table 3. Although the number of live births during the two protocol periods differed by nearly a factor of six, the overall incidences when corrected for the number of cases per 1000 live births differed greatly. It is, however, too early to state conclusively whether this was due to the different protocols employed or to other factors.

Various types of treatment were applied in the cases of ophthalmia neonatorum examined in this series, ranging from normal saline irrigation alone to intravenous penicillin and topical antibiotic drops. The treatment given was dependent on the type and severity of infection diagnosed. Sulphafurazole eyedrops were the most often used treatment. They were also the primary treatment in SON and OON, the two most frequently occurring causes of ophthalmia neonatorum in our study.

\section{Discussion}

Greenberg and Vandow, in a review of ophthalmia neonatorum in New York City during the 1950s, found no cases of GON when IM penicillin was used as prophylaxis. ${ }^{7}$ Furthermore IM penicillin has been found to be associated with a decreased incidence of early onset group B streptococcal disease. ${ }^{3}$

In more recent studies however, the changing picture of ophthalmia neonatorum has become apparent. The emergence of penicillinase-producing gonorrhoea (PPNG) and the increasing incidence of ChON show the need for implementation of better prophylaxis, diagnosis, and treatment. ${ }^{168}$

Chlamydia has been found in one study to cause nearly $50 \%$ of neonatal conjunctivitis. ${ }^{9}$ Inclusion conjunctivitis, once considered benign and selflimited, has now been shown to cause micropannus, conjunctival scarring, and later recurrence, which can be prevented by local application of tetracycline before the twelfth day of life. ${ }^{5}$ Both penicillin and silver nitrate are ineffective against chlamydia.

Because of the changing nature of the pathogens causing ophthalmia neonatorum MSH has recently updated its protocol. ${ }^{10} \mathrm{We}$ continue to give IM penicillin to prevent infection by the more prevalent penicillin-sensitive strains of $N$. gonorrhoeae and group B streptococcal disease. In addition since 1982 we have instilled $1 \%$ tetracycline ointment into the eyes of all newborn babies to eliminate the threat of PNGON and ChON.

Little controversy exists as to the benefit of prophylaxis in preventing ophthalmia neonatorum, especially GON. The debate has mainly centred on the choice of the agent which is the most effective and least toxic. Table 4 presents comparison studies based on both incidence of positive cultures and the occurrence of chemical conjunctivitis with different

Table 4 Ophthalmianeonatorum: percentage (\%) incidence with different prophylactic agents

\begin{tabular}{|c|c|c|}
\hline Investigator & Silver nitrate & Other agents \\
\hline Jarvis et al. ${ }^{*} \dagger$ & - & $\begin{array}{l}0.31 \text { Overall } \\
0.33 \text { IM penicillin } \\
0.13 \text { IM penicillin and } \\
\text { tetracycline }\end{array}$ \\
\hline Stenson et al. $\dagger^{8}$ & - & $0 \cdot 30$ Overall \\
\hline Armstrong et al. $\dagger^{1}$ & $\begin{array}{l}0 \cdot 19 \\
0 \cdot 86\end{array}$ & 0.38 Tetracycline \\
\hline Christian $\dagger^{11}$ & $1 \cdot 3$ & 0.68 Erythromycin \\
\hline Jarvis et al. ${ }^{*} \ddagger$ & - & $\begin{array}{l}0 \cdot 07 \text { Overall } \\
0 \cdot 008 \text { IM penicillin } \\
0 \text { IM penicillin and } \\
\text { tetracycline }\end{array}$ \\
\hline Greenberg and Vandow $\ddagger^{7}$ & $0 \cdot 03$ & $\begin{array}{l}0 \cdot 36 \text { Bacitracin } \\
01 \mathrm{M} \text { penicillin }\end{array}$ \\
\hline Posner et al. $\ddagger^{12}$ & $0 \cdot 20$ & Bacitracin \\
\hline${\text { Bickel } \ddagger^{13}}$ & $0 \cdot 15$ & OSulphacetamide \\
\hline Franklin $\ddagger^{14}$ & $0 \cdot 13$ & 0 IM penicillin \\
\hline Christian§"1 & $8 \cdot 7$ & 3.4 Erythromycin \\
\hline Franklin $\S^{14}$ & $6 \cdot 0$ & $2 \cdot 1$ IM penicillin \\
\hline Harris $\$^{16}$ & $3 \cdot 4$ & $2 \cdot 5$ IM penicillin \\
\hline Mathieu $\S^{17}$ & 46 & 6.0 Oxytetracycline \\
\hline Wachter et al. $\S^{18}$ & 39 & 16 Erythromycin \\
\hline Margileth $\S^{19}$ & 50 & $\begin{array}{l}1.0 \text { IM penicillin } \\
0.7 \text { Bacitracin }\end{array}$ \\
\hline
\end{tabular}

*Excludes four cases born elsewhere. $†$ All ON cases. $¥$ GON only. §Chemical conjunctivitis only. 
Table 5 Ophthalmia neonatorum: comparison of incidence and aetiological breakdown

\begin{tabular}{|c|c|c|c|c|c|}
\hline \multirow[b]{2}{*}{$\begin{array}{l}\text { Incidence } \\
\text { (cases per } 1000 \\
\text { live births) }\end{array}$} & \multicolumn{2}{|c|}{ Jarvis etal.* } & \multicolumn{2}{|l|}{ Stenson et al. ${ }^{8}$} & \multirow{2}{*}{$\begin{array}{l}\text { Armstrong etal. }{ }^{1} \\
\\
\text { Silvernitrate } \\
8 \cdot 6\end{array}$} \\
\hline & $\begin{array}{l}\text { Penicillin } \\
3 \cdot 33\end{array}$ & $\begin{array}{l}\text { Penicillin and } \\
\text { tetracycline } \\
1 \cdot 33\end{array}$ & $\begin{array}{l}\text { Silver nitrate } \\
1.9\end{array}$ & $\begin{array}{l}\text { Tetracycline } \\
3 \cdot 8\end{array}$ & \\
\hline Gonococcal (\%) & $2 \cdot 3$ & 0 & $2 \cdot 3$ & $4 \cdot 7$ & $14 \cdot 2$ \\
\hline Gonococcal and chlamydial (\%) & 0 & 0 & N/A & N/A & $1 \cdot 0$ \\
\hline Chlamydial (\%) & 0 & 0 & $33 \cdot 0$ & $31 \cdot 0$ & $28 \cdot 5$ \\
\hline Staphylococcal (\%) & $31 \cdot 8$ & $17 \cdot 0$ & N/A & N/A & $10 \cdot 3$ \\
\hline Chemical $(\%)^{*}$ & $5 \cdot 9$ & $33 \cdot 0$ & N/A & N/A & $1 \cdot 6$ \\
\hline Uncertain (NON and UON) (\%) & 34 & $17 \cdot 0$ & $30 \cdot 0$ & $27 \cdot 0$ & $44 \cdot 4$ \\
\hline Bacterial (\%) & $57 \cdot 6$ & $50 \cdot 0$ & $37 \cdot 0$ & $42 \cdot 0$ & N/A \\
\hline
\end{tabular}

*Excludes four cases born elsewhere. †Definitions of chemical conjunctivitis differ slightly.

prophylactic agents. Stenson $e t$ al. found an overall percentage incidence of ophthalmia neonatorum of 0.30 cases, representing 3.0 cases per 1000 live births. ${ }^{8}$ Armstrong et al. found an overall incidence of 8.6 cases per 1000 live births $(0 \cdot 86 \%$ incidence $){ }^{1}$ Christian found percentage incidences of 1.3 and 0.68 for silver nitrate and erythromycin prophylaxis respectively. " Our study found a percentage incidence similar to that reported by Stenson, namely, $0 \cdot 31$, representing $3 \cdot 1$ cases per 1000 live births. Under our earlier protocol of IM penicillin alone we found a $0.33 \%$ incidence, representing 3.3 cases per 1000 live births. Using the combined protocol of IM penicillin and topical tetracycline, we found a percentage incidence of $0 \cdot 13$, representing 1.3 cases per 1000 live births. These figures demonstrate a significantly small incidence while using both MSH prophylactic protocols.

Two studies of comparable design to ours are outlined in Table 5. As in our series Stenson et al. ${ }^{8}$ compared two different prophylactic regimens and found 1.9 and 3.8 cases per 1000 live births for silver nitrate and tetracycline respectively. MSH protocols produced rates of 3.33 and 1.33 cases per 1000 live births for IM penicillin and IM penicillin with topical tetracycline respectively. Of all the studies cited, the MSH combined prophylaxis protocol resulted in the smallest ophthalmia neonatorum rate $(1.33$ cases per 1000 live births).

Our study also shows GON rates that are among the lowest of the studies cited in Table 4. In our 10year series three cases of GON were found, in one of which the infant was born elsewhere. Hence two cases actually occurred under MSH protocol, while 30345 live births were recorded here. This represents an overall incidence of 0.07 cases per 1000 live births $(0.007 \%$ incidence) and 0.08 cases per 1000 live births $(0 \cdot 008 \%$ incidence) for IM penicillin alone, and no cases for IM penicillin combined with topical tetracycline. This is equalled by Greenberg et al., Posner et al., ${ }^{12}$ Bickel, ${ }^{13}$ and Franklin ${ }^{14}$ using the various forms of prophylaxis shown on Table 4.

The GON results summarised in Table 5 further reinforce the efficacy of the later MSH protocol as compared with others. All the ChON cases found in our series were born elsewhere. No cases occurred under either of the MSH protocols for the 10-year period. As discussed earlier, chlamydia can cause up to $50 \%$ of neonatal conjunctivitis. ${ }^{9}$ Armstrong et al. ${ }^{1}$ found chlamydia to be the prime cause of ophthalmia neonatorum in those cases where a cause was found. In their series it caused $28.5 \%$ of cases. Stenson et al. ${ }^{8}$ also found chlamydia as the cause of ophthalmia neonatorum in a large percentage of cases, $33 \%$ and $31 \%$ for silver nitrate and tetracycline respectively. The disparity between these figures and ours is probably due to lack of experience at this institution with ChON. Only three cases have been diagnosed in the past 10 years.

An additional explanation for the disparity may be the fact that the importance of ChON has only recently been realised. Our study is retrospective, relying on notations in charts made up to 10 years ago. Often conjunctival scrapings were not performed, and when performed were not collected, stained and read properly. Moreover, the unknown group in our study is large, and may harbour undiagnosed $\mathrm{ChON}$ cases.

In our series the $\mathrm{SON}$ and $\mathrm{OON}$ groups represent a large percentage of all cases of ophthalmia neonatorum. SON accounted for $30.2 \%$ of the 95 diagnosed cases in this study, while Armstrong et al.' found staphylococcus to be the cause of only $10 \cdot 3 \%$ of cases. Comparing our results with those of Stenson et al. ${ }^{8}$ who found about $40 \%$ (37 and 42) for all bacteria, we found that about $55 \%$ of cases at MSH to be bacterial in nature (see Tables 1 and 5). This is similar to Dumont and DeHaze's findings as well. ${ }^{15}$

The uncertain group (NON and UON in our study) showed percentages that were nearly equal for the three studies outlined in Table 5 . In our series the uncertain cases represented $31.5 \%$ of all cases of 
ophthalmia neonatorum, which is somewhat disappointing.

The overall percentage incidence for the $\mathrm{CC}$ group in our study is 0.02 or 0.2 cases per 1000 live births, which is significantly smaller than the $\mathrm{CC}$ studies presented in Table 4. Although there is a large increase in CC in our study with the addition of topical tetracycline to our protocol, the incidence is only $0 \cdot 4 / 1000$ live births $(0 \cdot 04 \%$ incidence $)$, which is again significantly less than that represented in the CC studies outlined in Table 4. However, Armstrong et al. found only $1.6 \%$ of ophthalmia neonatorum to be chemical in nature.' Their definition, however, was different from ours, namely, the appearance of symptoms within 24 hours with negative smears and cultures. Our category was defined to include the appearance of symptoms within 36 hours with normal flora.

Armstrong et al. ${ }^{\prime}$ examined similar clinical characteristics in their study of 302 cases of ophthalmia neonatorum occurring at Grady Memorial Hospital from 1967 to 1973, and found certain clinical characteristics to be statistically significantly different, while our study did not. They found (1) a significant seasonal variation of GON and ChON; (2) that $\mathrm{ChON}$ occurred significantly more frequently in blacks than in whites; (3) that SON occurred more often in males $(71 \%)$ than females but not significantly; and that the ChON incubation period of $8 \cdot 1$ days was significantly longer than the 6.5 day period for GON.

As stated earlier, our series revealed no statistically significant differences among the groups, including incubation periods. The type of discharge was characterised as purulent in $23 \%$ of cases in our series and $81 \%$ by Armstrong et al. ${ }^{\prime}$ Fever was found in that study in only $9 \%$, while we experienced it in $5 \%$ of cases. Armstrong et al. ${ }^{1}$ also state that no uniform therapy for SON was found. In our series SON was treated with sulphafurazole eye drops in $45 \%$ of cases and OON treated as such in $58 \%$ of cases. Neither study reported ocular sequelae in its respective series.

\section{CONCLUSIONS}

Ophthalmia neonatorum remains an active clinical problem, probably with recent significant changes in its aetiology. Our series, however, is not consistent with this picture. In contrast with other studies, chlamydia did not prove to be a major cause of ophthalmia neonatorum at MSH. In addition a large percentage of cases $(31.5 \%)$ in our series was without an aetiological diagnosis. These results emphasise the need for improved diagnostic efficiency. This may be accomplished by earlier referral before antibiotics are used and by the increased availability of culture and serological techniques to identify chlamydia.
The wide range of clinical characteristics investigated in our series did not reveal statistically signifcant differences between the aetiological categories. Of further benefit to the clinician would be a study examining whether these clinical characteristics are different for the entire ophthalmia neonatorum group as compared with normal neonates.

The decade of experience with ophthalmia neonatorum at the Mount Sinai Hospital has proved our prophylactic measures to be efficacious. The current protocol of IM penicillin and topical tetracycline thus far seems particularly advantageous, but further evaluation will be necessary to establish this conclusively.

\section{References}

1 Armstrong JH, Facarias F, Rein MF. Ophthalmia neonatorum: a chart review. Pediatrics 1976; 57: 884-92.

2 Mooney BR. Gonococcal ophthalmia neonatorum prophylaxis: ointment vs. liquid. Infect Control 1984; 5: 138.

3 Steigman AJ, Bottone EJ, Hanna BA. Intramuscular penicillin administration at birth: prevention of early-onset group B streptococcal disease. Pediatrics 1978; 62: 892-3.

4 Raucher HS, Newton MJ, Fedultowicz H. Ophthalmia neonatorum caused by penicillinase-producing Neisseria gonorrhoeae. Pediatrics 1982; 100: 925-6.

5 Gutman LT. Chlamydia. In: Joklik WK, Willett HP, Amos DB, eds. Zinsser microbiology. 17th ed. New York: AppletonCentury-Crofts, 1980: 949-50.

6 Raucher HS, Newton MJ. New issues in the prevention and treatment of ophthalmia neonatorum. Ann Ophthalmol 1983; 15: $1004-9$.

7 Greenberg M, Vandow JE. Ophthalmia neonatorum: evaluation of different methods of prophylaxis in New York City. Am J Public Health 1961; 51: 836-45.

8 Stenson S, Newman R, Fedukowicz A. Conjunctivitis in the newborn: observations on incidence, cause, and prophylaxis. Ann ophthalmol 1981; 13: 329-34.

9 Forster RK. Ophthalmia neonatorum. In: Duane TD, Jaeger EA, eds. Clinical ophthalmology. Philadelphia: Harper and Row, 1978; 4: ch. 6: 1-9.

10 Brown EG. Maternal and child health labor and delivery policy manual. Policy No. 32. Newborn Medication Administration in Labor and Delivery Suites. 24 Feb, 1982.

11 Christian JR. Comparison of ocular reactions with the use of silver nitrate and erythromycin ointment. Ophthalmia neonatorum prophylaxis. J Pediatr 1960; 57: 55-60.

12 Posner AC, Anderson GD, Prigot A. Observations on prophylaxis of ophthalmia neonatorum in a municipal hospital. Antibiotics Annual 1958-9:134-7.

13 Bickel JE. Sodium sulfacetamide for prophylaxis of gonorrheal ophthalmia neonatorum. J Pediatr 1950; 37: 854-61.

14 Franklin HC. Prophylaxis against ophthalmia neonatorum. JAMA 1947; 134: 1230-5.

15 Dumont P, DeHaze M. Ophthalmia neonatorum. Brux Med $1955 ; 35: 2125-37$.

16 Harris ST. Penicillin versus silver nitrate in ophthalmia neonatorum prophylaxis. Sightsaving 1957; 27: 152-3.

17 Mathieu PL. Comparison study: silver nitrate and oxytetracycline in newborn eyes. J Dis Child 1958; 9: 609-11.

18 Wachter H, Pennoyer MM. Prophylaxis in the eyes of newborn infants. Mo Med 1956; 53: 187.

19 Margileth AM. Comparison of ocular reactions using penicillin and bacitracin ointments in ophthalmia neonatorum prophylaxis. J Pediatr 1957; 51: 646-51.

Accepted for publication 3 July 1986. 